any irreducible (that is, not factorizable) polynomial equation in two variables, of genus $\mathbf{2}$ or more, could have only a finite number of rational solutions (Proc. Cambridge phil. Soc. 21, 179; 1922). He did so on the most slender evidence, and for a long time the conjecture was considered to be just one of those things that would make the world nicer if it were true - but could just as easily turn out to be false. For example, the finiteness result for Fermat's Last Theorem follows immediately, since the genus $1 / 2(n-1)(n-2)$ is at least 3 if $n \geq 4$, and the case $n=3$ was proved by Euler in about 1738. In 1968, however, the Russian mathematician A.N. Parshin (Math. USSR Izvestija 2, 1145; 1968) showed that Mordell's conjecture would follow from another conjecture due to I.R. Shafarevich, about the behaviour of equations under 'reduction modulo a prime', and that conjecture was more plausible.

Faltings has proved the Shafarevich conjecture - and hence also that of Mordell - by a massive mobilization of the available machinery of algebraic geometry augmented by some crucial new ideas of his own. Treading an established path, he generalizes the problem from curves to higher-dimensional objects known as abelian varieties. He introduces a measure of the complexity of such a variety, named its height, and finds a formula describing how the height changes if the variety is replaced by a different but similar one (resulting from a technical operation known as an isogeny). One implication of this formula is a conjecture due to J.Tate (Invent. Math. 2, 134; 1966) about mappings between abelian varieties.

The road to a finiteness result is now open: first, show that only a finite number of the relevant objects have a given height; and second, show that the heights themselves are bounded by some definite upper limit. For the Shafarevich conjecture the relevant objects turn out to be 'principally polarized abelian varieties of a given dimension having good reduction except for a given finite set of primes'. The finiteness is established using yet another series of conjectures, made by André Weil, and proved by Pierre Deligne in the late 1970 s.

Ian Stewart is at the Mathematics Institute, University of Warwick, Coventry CV4 7AL, Warwickshire.

\title{
Neurobiology
}

\section{Cross-section of the spinal cord}

\section{from Jennifer Altman}

The London Spinal Cord Club is unusual as its membership is based on an interest in the tissue investigated rather than the academic disciplines of the investigators. Its recent symposium ${ }^{*}$ reflected this in the wide range of approaches and techniques, from cDNA probes and voltage-clamp recording to studies on clinical patients.

Two very different conceptual approaches were in evidence: on the one hand a reduction of the system to its minimum, using tissue culture, tissue slice or isolated cord preparations for intracellular recording, and dealing with the physiology and pharmacology of single units; on the other hand, a move back towards studying populations of neurones in situ. The first approach is necessary for studying the membrane effects of putative transmitters (M. Mayer and G. Westbrook, NIH, Bethesda) and the interactions between drugs, transmitters and receptors (A. King et al., St Bartholomew's Hospital, London). Isolated cord preparations have also been used for examining changes in electrical properties of rat sensory neurones as they mature (B. Fulton, University College, London) and for identifying abnormal neurone functions in the spastic mouse mutant (T. Biscoe and M. Ducher, University College, London). Such studies do little, however, to advance our understanding of how the spinal cord

*'Recent advances in the spinal cord': a Brain Research Association symposium, held at the Centre for Neuroscience, University College London, 6- 7 July 1984. works as a system, integrating inputs to produce defined and precise motor outputs. It is here that the whole-animal investigations are essential, and modern neuroanatomical and neurochemical methods of tracing and mapping neuronal populations are proving powerful tools for this type of analysis.

Numerous populations of neurones can now be identified because they contain specific marker molecules, including enzymes and peptides. The development of cDNA probes for tissue-specific sequences (J. Dickson et al., Institute of Neurology, London), now in its infancy, promises to be an important tool for characterizing neurones. But the question of the functional significance of the markers remains open. A link between histochemistry and function has been found for glycogen phosphorylase (C. Woolf et al., University College, London), the active form of which is found only in stimulated neurones and therefore identifies active cells. However, in the case of peptides identified in primary afferents, there is still no good evidence that they have specific transmitter or modulator effects on postsynaptic cells, as has been shown for cholycystokinin in cortex (J. Kelly, St George's Hospital, London). The demonstration that developing motor neurone terminals contain $\beta$-endorphin, an opioid peptide, that disappears as the neurones mature but reappears when their axons are damaged (L. Haynes et al.. Birmingham Medical
School and National Institute of Medical Research, London), raises the possibility that some peptides could be left over from development or be involved in synaptic maintenance. Haynes et al. went on to show that $\beta$-endorphin has a neurotrophic effect on cholinergic transmission by regulating the type of acetylcholinesterase in the synaptic cleft .

The great advantage of the spinal cord over other parts of the central nervous system for studying neuronal integration is that the outputs can be monitored at the same time as inputs are manipulated. Between the inputs and the outputs, however, lie complex networks of interneurones, which are still relatively inaccessible to investigation. Nonetheless, E. Jankowska (Göteborg) has identified interneurones of particular reflex pathways using transneuronal transport of horseradish peroxidase conjugated with wheat germ agglutin. This work, together with results from $S$. McMahon (University College, London) on the distributions of $\mathrm{C}$ fibre terminals in skin and muscle and from S. Hunt (MRC Neuropharmacology, Cambridge) showing that cutaneous afferents containing substance $P$ terminate more dorsally than those marked by fluoride-resistant acid phosphatase, begins to establish a picture of functional subdivisions within the laminae of the cord.

Multiunit recording from neurone populations requires sophisticated statistical analysis and interpretation is very difficult. How significant is a small relatively rare event such as the monosynaptic input from bulbo-spinal interneurones to intercostal motor neurones (P. Kirkwood et al., Institute of Neurology, London)? Recordings from single identified motoneurones in the locust during the generation of rhythmic behaviour such as flight show that inputs of only $1-2 \mathrm{mV}$ can advance the neurone's firing providing they occur at the right time in the depolarization cycle (Robertson, R.M. \& Pearson, K.G. J. comp. Neurol. 215, 33; 1983). This observation supports Kirkwood's suggestion that spinal motoneurone outputs may be subtly influenced by quite small changes in the timing of the inputs.

Lastly, the presence of two paraplegic observers from the International Spinal Research Trust meant that the pathological applications of work on the spinal cord were kept in mind. Research into reflex pathways in humans, both normal subjects and patients with clinical disabilities (B. Day et al., Institute of Psychiatry, London; J. Iles and $R$. Roberts, Universities of Oxford and London; $T$. Davies, Guy's Hospital, London) is confirming results from animal studies and should lead to improved diagnosis, although the meeting had little immediate to offer towards the prevention or cure of spinal cord malfunction.

Jennifer Altman can be contacted at 7 Holebottom, Todmorden, Lancashire OL14 $8 D D$. 